I am indebted to Mr. O. H. Truman for reducing the measures and for computing the radial velocity corrections to the wavelengths.

Lowell Observatory,

V. M. SLIPHER.

Flagstaff, Arizona.

\title{
Stationary Sodium Lines in Spectroscopic Binaries ${ }^{1}$
}

From the nature of a spectroscopic binary it seems reasonable to expect that the velocities given by the separate star lines on a single plate should be in mutual agreement. This has been found to be the case normally, the displacements of the star lines on any one plate corresponding to the same velocity. However, the $\mathrm{H}$ and $\mathrm{K}$ lines of calcium occasionally offer an exception to this usual behavior. In a number of early type spectroscopic binaries these lines remain practically stationary thruout a periodic variation of large amplitude in the other star lines, and moreover, they appear sharp and narrow in contrast to the broad and hazy lines characteristic of this class of spectra.

A recent investigation at the Lick Observatory has shown a similar peculiarity in the $\mathrm{D}_{1}$ and $\mathrm{D}_{2}$ lines of sodium. Two stars, $\beta$ Scorpii and $\delta$ Orionis, were studied, these having been selected as representative of the class of variable velocity stars showing stationary $\mathrm{H}$ and $\mathrm{K}$ lines. The spectrograms which form the basis of this work were taken in the visual region with a three-prism spectrograph attached to the thirty-six-inch refractor. In this region of the spectrum but three oscillating lines were measurable and, due to their poor definition, the velocities determined from these are subject to large uncertainty. The $\mathrm{D}_{1}$ and $\mathrm{D}_{2}$ lines of sodium are quite sharp and better suited for exact measurement, $\mathrm{D}_{2}$ being uniformly better than $\mathrm{D}_{1}$. .

The results of the investigation are summarized below.

I. The D lines of sodium appear sharp and narrow on all the spectrograms, resembling $\mathrm{H}$ and $\mathrm{K}$ in this particular.

2. Within the limits of observational error these lines remain stationary thruout the period. A maximum departure of $2 \mathrm{I} \mathrm{km}$. from the mean velocity can be attributed to accidental error, since the character of the lines on some of the plates makes their measurement quite inaccurate and since in this region the dispersion is small.

1A detailed account of this investigation is given in Lick Obs. Bull., 326. 
3. A comparison of the mean velocity from the sodium lines with the mean velocity from the $\mathrm{H}$ and $\mathrm{K}$ lines, the velocity of the system, and the component of solar motion is presented in the following table:

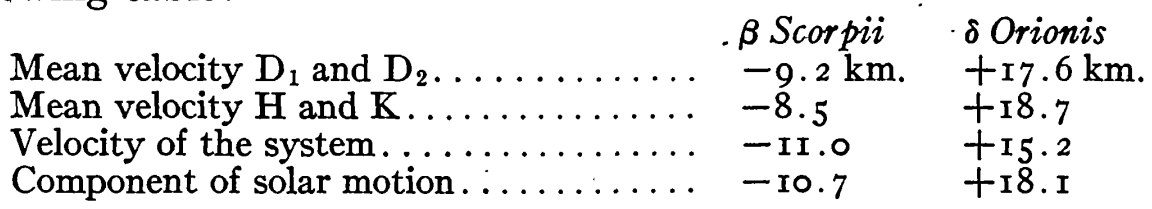

4. On account of the uncertainty of the values from the oscillating lines and also in consideration of the comparatively few observations that were obtained, no attempt was made to determine a new velocity curve. Within the limits of their accuracy, our observations are satisfied by the orbits as determined at the Allegheny Observatory.

The close relationship between the $\mathrm{D}$ lines of sodium and the $\mathrm{H}$ and $\mathrm{K}$ lines of calcium in the two stars which have been studied is very striking. Both in appearance and in behavior these lines are entirely comparable. We have still to look for an explanation of the peculiarity in these lines. The agreement of the velocity obtained from the $\mathrm{D}$ lines with both the component of solar motion and the velocity of the system seem to favor equally the two principal hypotheses which have been suggested to explain the behavior of $\mathrm{H}$ and $\mathrm{K}$; first, that the effect is caused by an absorbing cloud of calcium vapor stationary in space between the binary star and the observer; second, that its origin lies in some condition connected with the binary system, perhaps an envelop of calcium vapor around the system as a whole. These theories are as readily applicable to the $\mathrm{D}$ as to the $\mathrm{H}$ and $\mathrm{K}$ lines, but with no less uncertainty. At present it seems best merely to recognize the relation of the sodium and the calcium lines in the stars which have been studied and seek an explanation which will account for the peculiarity of both.

Mary Lea Heger.

\section{A Variable Star with a Peculiar Spectrum}

The long-period variable star R Aquarii 233815 has been found recently to possess a very peculiar spectrum. The data concerning this star are as follows: R. A. (I900) 23 hrs. $38.6 \mathrm{~min}$; Dec. (I900) - I $5{ }^{\circ} 50^{\prime}$; Magnitude, variable, 6.2 to II:0; Period 387.2 days; Predicted maximum r9r9 Nov. 22; Spectrum Md8. The following 